\title{
Development of a prototype for a prosthesis implant of one degree freedom for the ankle joint
}

\begin{abstract}
The manufacture of a joint ankle implant prostheses is one of the most relevant topics when it comes to improving the quality of life in patients who lose this movement motor function. Therefore, a design is required that emulates the flexo-extension movement as naturally as possible.
\end{abstract}

Keywords: ankle joint, prosthesis, manufacture, gait, support phases
Volume 3 Issue 5 - 2019

\author{
Hernández Ramírez A, Urriolagoitia- \\ Sosa G, Díaz León C, Romero Ángeles B, \\ Hernández Cerón A, Torres San Miguel C R, \\ Urriolagoitia Calderón $\mathrm{G}$ \\ Instituto Politécnico Nacional, México
}

Correspondence: Aldo Hernández Ramírez, Instituto Politécnico Nacional, Higher School of Mechanical and Electrical Engineering, Delegation Gustavo A. Madero, C.P. 07320, Mexico, Email alher9194@gmail.com

Received: October 15, 2019 | Published: October 30, 2019

\section{Introduction}

The loss of movement in the ankle joint is repeatedly linked to osteoarthritis, which is a disease that causes wear and tear on the cartilaginous tissue, preventing free movement between the tibia, fibula and astragalus. Currently one of the treatments to regain movement in the ankle joint is ankle arthrodesis, which is a surgical procedure during which flexo-extension mobility is restored to resume the high and low gait cycle Speed. To manufacture an artificial ankle joint it is necessary to establish the parameters that help control the manufacturing processes of each component, ensuring its proper operation. The mechanical design for this joint highly depends on the analysis of axial loads produced by the weight of the human body during the supporting phases and based on that information, select the appropriate materials that are useful not only to support the weight of the human body but maintaining an excellent level of biological compatibility.

\section{Methodology}

To establish a functional design that replaces the ankle joint, previous designs such as Hintegra, Star and Agility are taken into consideration, as they are relatively simple to manufacture and do not present a greater complexity when inserted into the ankle (Figure 1).
The parts shown below Figure 2 were manufactured from a polymer known as RenShape to obtain a prototype for its optimization and be able to visualize how it would be inserted inside the ankle. The main advantage within the prosthesis is that if any component were to fail it could be replaced very easily thanks to the simplicity of its design. When manufacturing any component of this type the most important consideration to have is not to alter the delicate balance between the functioning of the human body and the user's lifestyle.

The slightest oversight in relation to the weight of the components prosthesis would mean an addition of extra weight to the foot. This in the long term would lead to a condition dificulting the ability to maintain balance, and could lead to produce a poor posture in the spine. The most convenient manufacturing process to obtain the metal elements for this type of prosthesis is the implementation powder as raw material. The Ti-6Al-4V is excellent biological compatibility with the live tissue of the human body as well as its excellent resistance and a relatively light weight. In addition, a virgin PEAD high-density polyethylene with no dyes or chemicals that are toxic to the organism is used to obtain the insert. The way to obtain this piece is through its $\mathrm{CNC}$ (Computer Numerical Control) machining in which the machining commands must be prepared and loaded inside the machining center computer.

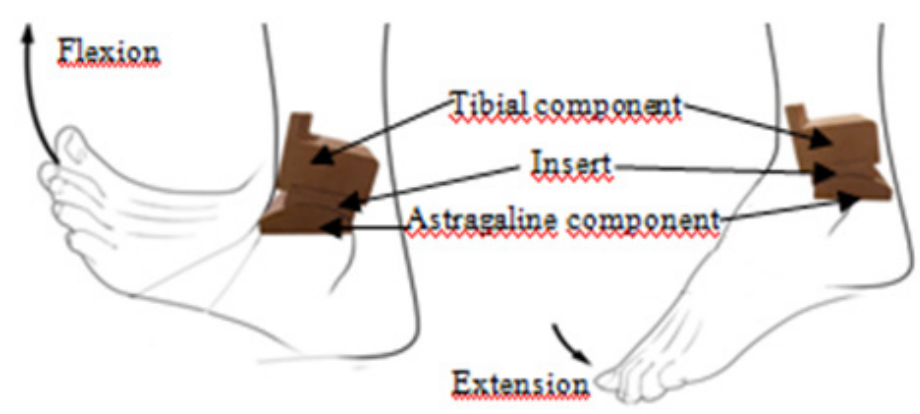

Figure I Design and assembly of the joint ankle prosthesis. 


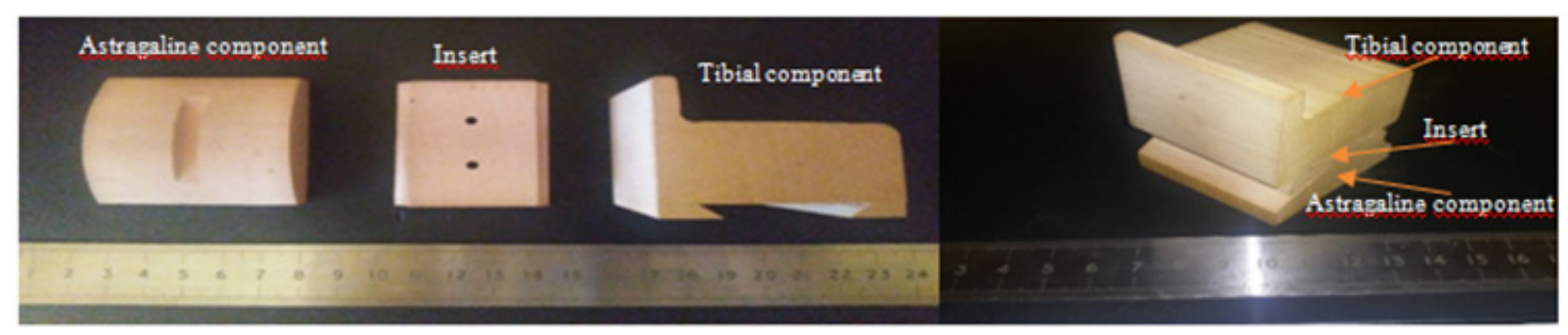

Figure 2 Manufacture of the joint ankle prosthesis prototype.

On the other hand, within this work it has been considered the numerical analysis corresponding to the assembly of the prosthesis with a load of $980.66 \mathrm{~N}$ which corresponds to a total weight of 100 $\mathrm{k}$ at the moment when a person is supporting the weight directly on the ankle in which the prosthesis is placed and on the other hand the elongation field is for whole prosthesis also appreciated Figure 3 and also it is shown the result obtained employing Von Mises (Figure 4).

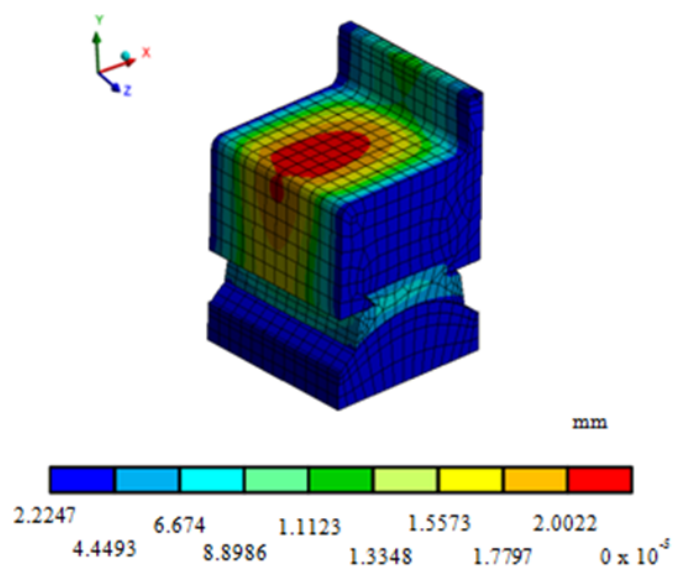

Figure 3 Total elongation of the ankle prosthesis $(\mathrm{mm})$.

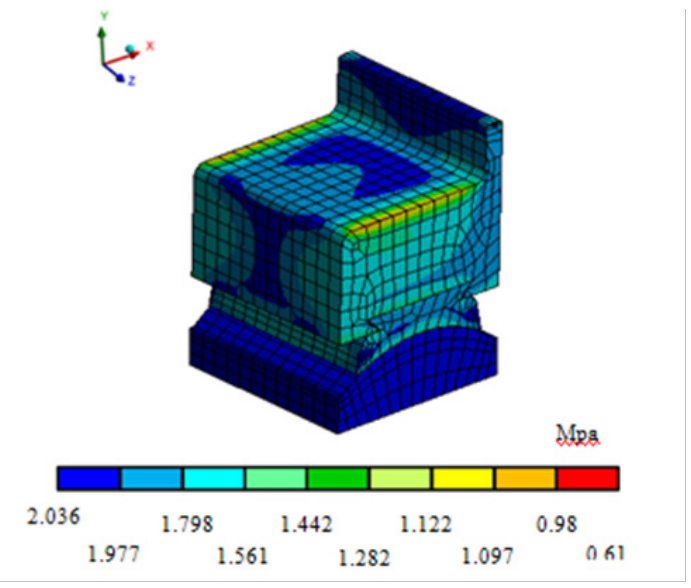

Figure 4 Von Mises equivalent stress of the ankle prosthesis.

\section{Discussion}

The manufacture of implant prostheses in particular for the ankle joint can be subjected to different changes or optimizations during its design. An example could be to increase the degrees of freedom or decrease the dimensions and volume of the metal components. It is certainly a prosthetic option that not only offers the aesthetics of a natural ankle but is also useful to help regain the user's confidence to walk. $^{1-3}$

\section{Conclusion}

Using finite element method, numerical analyses for the ankle prosthesis are established, in particular the results are displayed using total elongation and Von Mises with the latter to visualize that the material of the prosthesis can be work under the burden produced by the weight of the person without having the tendency to fail. Establishing a manufacturing process for implant prosthesis tends to be complicated as different parameters have to be met to ensure proper operation once it is inside the living tissue. On the other hand, it has to comply with the standards that monitor this type of products since on the one hand the materials must be biocompatible so as not to contaminate the living tissue and not affect the health of people and also necessary to respect the manufacturing conditions for each type of component. It should be mentioned that the manufacture of stents is maintained subject to the fact that each prosthetic device must be made in a personalized way for each user, because there will always be differences in bone dimensions in the lower limb. Another very important issue to consider, for the manufacture of this prosthesis, is that with the application of the powder metallurgy process, it is even possible to control the grain size of the part that is produced and this in turn generates the ability to manipulate the quality of absorption of cyclic axial loads, produced during the support phases on the gait.

\section{Acknowledgements}

The authors thank the Intituto Politécnico Nacional and the Cnnsejo Nacional de Ciencia y Tecnología for the support provided for the realization of this work.

\section{Conflicts of interest}

The author declares that there is no conflict of interest.

\section{Funding details}

None. 


\section{References}

1. Benirschke SK, Meinberg E, Anderson SA, et al. Fractures and dislocations of the midfoot; Lisfranc and Chopart injuries. J Bone Joint Surg Am. 2012;94(14):1325-1337.

2. Pedro JA, López Bravo A, San Román J. Experimental study of acrylic bone cements for orthopedic surgery reduced toxicity. Mapfre Medicina. 2002;13(3):155-164.

3. Devesa F, Amigó V, Benavente E, et al. nfluence of the percentage of Fe on the mechanical properties of Ti-25Nb-xFe alloys obtained by powder metallurgy, III National Congress of Powder Metallurgy. Valencia. 2010;13:505-514. 